\title{
Semantic Virtual Factory supporting interoperable modelling and evaluation of production systems
}

\author{
Botond Kádára(2), Walter Terkaj ${ }^{b}$, Marco Sacco ${ }^{b}$ \\ ${ }^{a}$ Computer and Automation Research Institute, Hungarian Academy of Sciences (MTA SZTAKI), Fraunhofer PMI PC, Kende u. 13-17, H-1111 Budapest, Hungary \\ ${ }^{b}$ Istituto Tecnologie Industriali e Automazione, Consiglio Nazionale delle Ricerche (ITIA CNR) via Bassini 15, 20133, Milano, Italy
}

Modelling, simulation and evaluation of manufacturing systems are relevant activities that may strongly impact on the competitiveness of production enterprises both during the design and the operational phases. This paper addresses the application of a semantic data model for virtual factories to support the design and the performance evaluation of manufacturing systems, while exploiting the interoperability between various Digital Enterprise Technology tools. The paper shows how a shared ontology-based framework can be used to generate consistent 3D virtual environments and discrete event simulation models, demonstrating this way how the proposed solution can provide an interoperable backbone for heterogeneous software tools.

Modelling, Simulation, Virtual Factory

\section{Introduction}

One of the main challenges in manufacturing today is to design and operate systems producing a high variety of customized products as efficiently and quickly as possible, while dealing with uncertain and highly volatile demands. Managing manufacturing companies and systems requires both long-term and short-term decisions, which all deeply influence the performance of these firms. From strategic point of view the decisions have impact on longer time horizon (usually more than two years) and involve major commitment of financial resources [1]. For instance, strategic decisions may regard the number of plants or facilities to be built, their size and their location, the variety of products to be manufactured, the appropriate manufacturing technologies, and, within a plant, the number and types of production resources, the characteristics of the transportation and handling systems and the degree of automation, to mention the most important aspects only. From tactical and operational points of view the decision makers should consider changes like expansion, reduction or reconfiguration of production structures, mid-term planning, short-term scheduling and optimized control of the systems in question. On all levels of hierarchy the complexity of these decisions and their importance from the point of view of the profitability emphasizes the need to have formal and structured approaches to support the design, management and performance evaluation of production systems.

Nowadays managers and engineers usually apply Digital Enterprise Technologies (DET) as decision support tools in handling the challenges enumerated above [2]. The concept of digital enterprise - the mapping of the key data and processes of an enterprise to digital structures by means of information and communication technologies - gives a unique opportunity for planning and controlling the operation of production systems [3]. Although DET provide all the necessary components for modelling, analysis and evaluation of production systems, in real scenarios these tools and solutions are concurrently used by different decision makers with various objectives. Mainstream commercial Digital Factory (DF) tool providers already offer integrated solution of their modular products but, on the one hand, the procurement and deployment of such integrated solutions are severely expensive and usually without the warranty to effectively exploit all provided functionalities, on the other, they requires specific knowledge and expertise frequently inaccessible even in big enterprises.

This paper introduces a research work aiming at homogenizing the modelling basis of production system and on this normalized foundation allowing the smooth interoperation of different DF tools that can be both commercial and self-developed.

\section{Modelling production systems on semantic basis}

Production system modelling may use different formalisms and approaches, depending on the characteristics of the considered problem and the expected results. Whether the system is a machine tool, a production line, a distribution network or a communication system, we can use modelling for formalizing and gaining knowledge from the system at different life-cycle phases, for evaluating a certain feature, for making comparison between several reconfiguration options, for problem detection or for evaluating and improving the system performance.

Formal, descriptive modelling methods proposed in Enterprise Engineering (EE) discipline [4] support the analysis and reengineering (design) of existing (new) business entities - namely business processes, application systems, business departments, industrial plants and in the broadest sense, complete enterprises or networks of enterprises. Descriptive methods like UML or IDEFxxx [5] consist of modelling languages, defined in their syntax and usually in correspondent graphical notations. In the most complete cases, formal methods provide a reference architecture where the supported modelling languages are organized according to pre-defined criteria (e.g. GERAM, ARIS) [6], [7].

\subsection{Standardization efforts}

From standardization point of view, several contributions have faced the problem of developing a holistic and complete data model for representing manufacturing systems, both considering 
tangible (e.g. machine tool, workpiece to be produced, etc.) and intangible (e.g. process plans, production logics, etc.) aspects.

ANSI/ISA-95 is an international standard for developing an automated interface between enterprise and control systems. It aims at providing both consistent terminology and information models as well as reliable operations models [8]. A different approach in the modelling of manufacturing process is offered by the Process Specification Language (PSL) standard. PSL is an ontology providing a way to formally describe a process and its characteristic. The ontology has been developed at the National Institute of Standards and Technology (NIST) and has been approved as an international standard in the document (ISO 18629) [9].

Partially based on Standard for the Exchange of Product model data (STEP), the Industry Foundation Classes (IFC) represents an open specification for Building Information Modelling (BIM) data that is exchanged and shared among the various participants in an architecture, engineering and construction project [10].

\subsection{The role of ontologies in production systems modelling}

Several research efforts were already made to apply semantics and create ontologies aiming at modelling the components and the interconnections in manufacturing systems and/or supporting the simulation model building of such systems. In [11] the study introduces a component-based modelling and simulation approach that supports model reuse across multiple application domains called CODES. The attributes and behaviour of the components are abstracted as meta-components and are described using COML (COmponent Markup Language). The integrated approach is supported by a component-oriented simulation and modelling ontology called COSMO. Authors in [12] presents the main issues and challenges of creating a simulationbased modelling ontology. On the base of the experience coming from the creation of the Discrete event Modeling Ontology (DeMO) the authors propose the decomposition of the models in behavioural and observable parts on the base of Hidden Markov Models. An overview of the DeMO ontology is also given, focusing on the semantic modelling of a discrete-event simulation kernel including both event-driven and process oriented approach.

In [13] the authors explain the role of ontologies in facilitating simulation modelling and highlighting the importance of integrating and modularizing different simulation systems. Special view is taken on the process of simulation model creation and the authors underline the most relevant project phases where ontology supports the common understanding. Further possible roles of ontological modelling are also introduced like distributed and component-based simulation. Finally the authors present the Ontology driven Simulation Modelling Framework (OSMF) solution, which provides a "visual programming environment" to rapidly compose, build, and maintain distributed, federated simulations

The research presented in [14] focuses on reusability and composability aspects in simulation modelling of large-scale systems independently from the type of simulation.

On the base of our review it was clear that semantic web technologies, even if promising, were only partially used in different, well-bordered segments of production systems' modelling field. Inspired by this fact and the supplementary knowledge manipulation opportunities provided by ontologybased modelling, the European FP7 Virtual Factory Framework (VFF) [15] project aimed at creating a new framework based on semantic web technologies to manage the data and models related to the whole factory life.

\section{The Virtual Factory Framework}

The Virtual Factory Framework (VFF) can be defined as "An integrated collaborative virtual environment aimed at facilitating the sharing of resources, manufacturing information and knowledge, while supporting the design and management of all the factory entities, from a single product to networks of companies, along all the phases of the their life-cycles" [16].

As presented in Figure 1, the VFF architecture is based on three main pillars:

- Virtual Factory Data Model (VFDM), i.e. a coherent, standard, extensible, and common data model for the representation of factory objects related to production systems, resources, processes and products, i.e. the Data \& Knowledge.

- Virtual Factory Manager (VFM), i.e. the software application that manages and provides access to the shared repository containing data structured according to the VFDM. A prototype implementation of VFM as web-service is presented in [18].

- Digital Factory tools (or VF modules), i.e. the software tools that are able to communicate with the VFM to retrieve and send shared data formalized according to the VFDM. Specific VF modules (e.g. Factory Image in Figure 1) may access real factory data to synchronize the real and virtual representations.

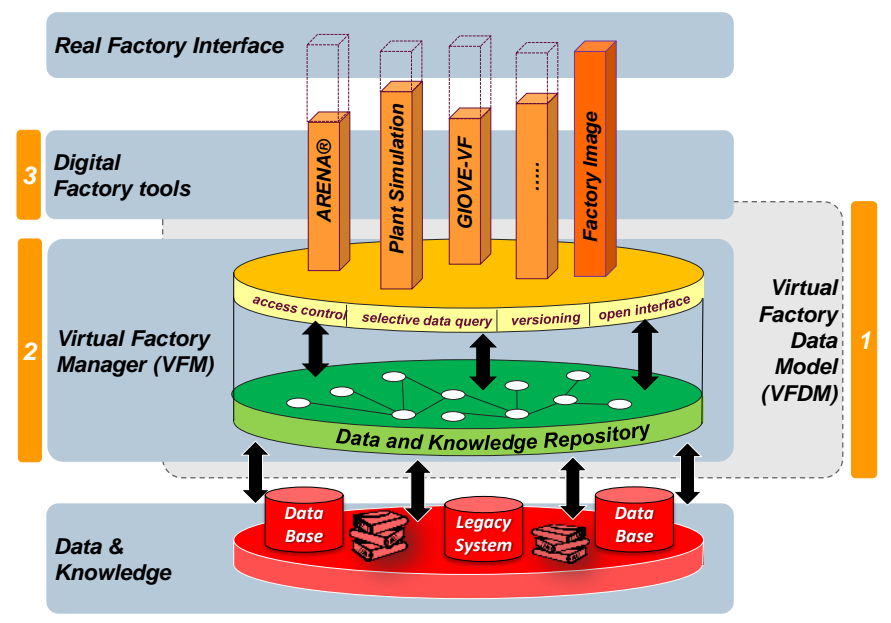

Figure 1. The architecture of the Virtual Factory Framework

As an integrated semantic data model VFDM implements and extends the core aspects of the IFC standard [10] to provide the base for integration of various digital tools applied in different planning and operation life-cycles phases of a manufacturing system. The extensions, partly based on ISA-95 standard, were developed to represent the characteristics of a manufacturing system in terms of the products to be manufactured, the manufacturing process they must undergo and the resources entitled to operate the different manufacturing operations.

The VFDM is decomposed into a hierarchical structure of ontologies, thus downsizing the problem and its complexity while keeping a holistic approach. Indeed, the VFDM was designed as a set of related ontologies [17] serialized as Web Ontology Language OWL) files according to Resource Description Framework (RDF/XML) [19]. The VFDM defines only the socalled meta data (i.e. the classes, properties and restrictions), whereas the actual instances (i.e. the individuals) are stored in the data and knowledge repository (Figure 1). As an overview, the following main areas are covered by the ontology classes defined in the VFDM:

- Product, modelling the data related to the product, i.e. the production goal of the factory.

- Resource, modelling the data related to the production resources that are used by a system with the final goal of 
transforming the product (or a work in progress). These resources can be human operators, machines, transportation and logistics related devices, etc.

- Process, modelling the data regarding the processes that are adopted by the system to directly (e.g. manufacturing system, assembly system) or indirectly (e.g. logistic processes, maintenance processes) transform a product.

- System, modelling the data of a transformation system (e.g. manufacturing, assembly, transportation and manipulation systems) that affects a product by means of physical resources and/or human resources within a process.

- Building, modelling the data related to the physical structure of the factory and important for 3D layout planning and visualisation aspects (e.g. walls, columns, floor, power supply lines, etc.).

Further details about the VFDM with a comprehensive description of relations between classes and properties are described in [20].

\section{VFF-based integration of Digital Factory tools}

This section delves into the problem of integrating a Digital Factory tool into the VFF, by developing a software layer named Virtual Factory Connector (VfConn) that takes care of Input/Output conversions from data stored in the shared repository to the internal data structures of the Digital Factory tool, and vice-versa. A proper VfConn may be developed only if the following fundamental requirements are met:

- The developer knows both the VFDM and the specific data model adopted by the Digital Factory tool, at least those parts that are relevant for supporting the data exchange.

- The Digital Factory tool offers a way to access and modify (if needed) its internal data structures, typically an application programming interface (API).

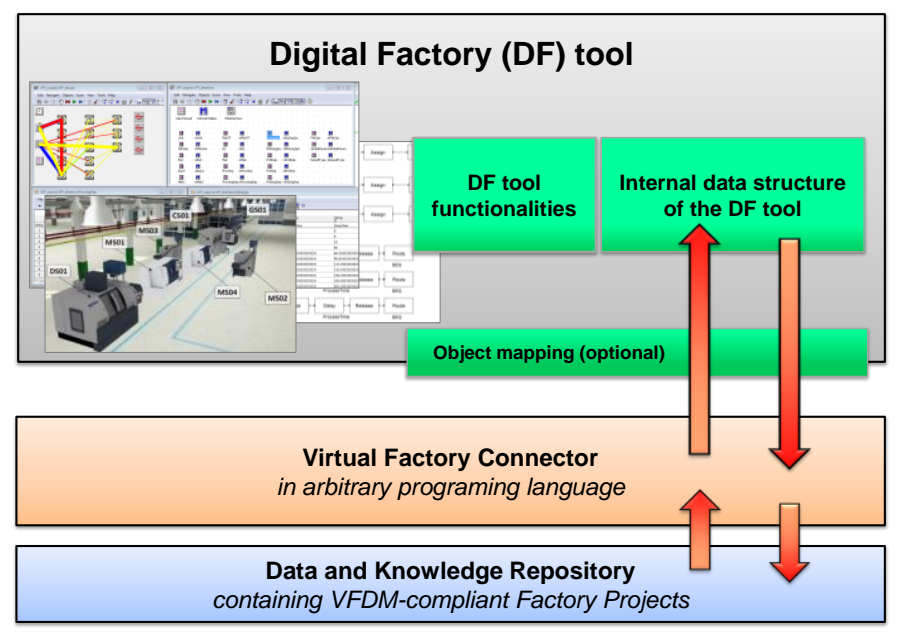

Figure 2. Integration of a new Digital Factory tool in VFF

If such requirements are met, then a Digital Factory tool can be integrated into VFF by realizing the following steps:

1. The classes and properties of the VFDM must be mapped to the data model adopted by the Digital Factory tool. If necessary, the VFMD can be extended by adding domain ontologies that formalize application-specific concepts.

2. Development of the VfConn by adopting the best feasible option according to the available technologies. Depending on the language required by the API of the digital tool, different programming libraries can be exploited to support the development of the VfConn.
3. Population of the shared repository with data and knowledge required as input by the newly integrated Digital Factory tool. Such population can be accomplished by means of Graphical User Interface (GUI) tools, by transferring data from existing databases or legacy systems, or by integrating further digital tools within VFF.

Figure 2. shows how a DF tool can be integrated in VFF thanks to a specific VfConn, while referring to the common VFDM. The functionalities of the DF tool can be used during the creation, modification of data and relations about the model of the system in question.

The following sub-sections address how two Digital Factory tools were integrated into VFF and can be employed to concurrently create and evaluate factory projects while sharing consistent data. Specifically, a 3D layout design tool and a commercial discrete-event simulation tool will be presented and then applied to a common industrial case representing a production line, as described in [22]. This production line consists of seven machine tools, characterized by failure modes that have to realize a part type by executing a process plan decomposed into five stages that include a drilling, two sequential milling, a quality control and a grinding operation.

\subsection{Production system design and visualization}

The production line of the industrial case can be designed and placed in its building by using 3D software tools, like for instance GIOVE Virtual Factory (GIOVE-VF) [21]. GIOVE-VF is a 3D virtual reality collaborative environment aimed at supporting the factory layout design. In particular, GIOVE-VF offers the user the possibility to design factories by selecting machines, operators and other resources from available catalogues and place them in the 3D scene of the virtual factory (Figure 3). The virtual environment can schematically display performance measures that are provided by simulators and/or monitoring tools.

GIOVE-VF has been developed in $\mathrm{C}++$ and can import/export ontologies serialized in RDF/XML format thanks to a specific VfConn that was developed using a $\mathrm{C}++$ library named VfConnectorLibCpp providing functionalities to parse, create and modify the ontologies by exploiting an internal map between OWL classes/restrictions and $\mathrm{C}++$ classes/methods. The VfConnectorLib library is based on the Redland C libraries and makes use of an in memory RDF storage.

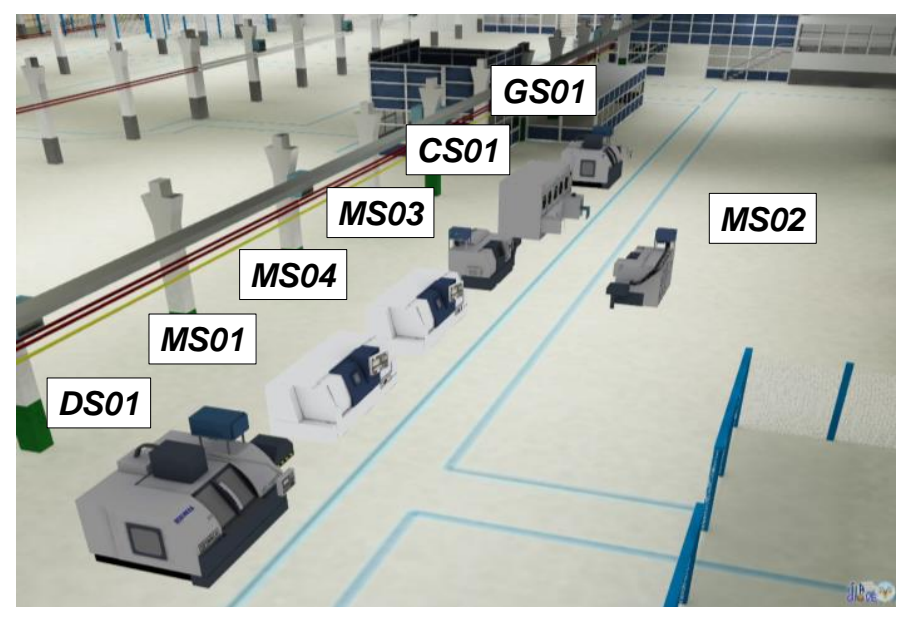

Figure 3. 3D representation of the test-case in the GIOVE-VF module

\subsection{Production simulation with a commercial DF tool}

Not only self-developed but also commercial DF tools can interoperate thanks to the VFDM while designing a production 
system. The commercial off-the-shelf simulation package Plant Simulation by Siemens PLM was also integrated in VFF to support the performance evaluation of production systems. Figure 4 shows the similar test-case that was designed in the GIOVE 3d virtual tool. A standard VfConn was designed and implemented in Java language to easily retrieve and save factory projects from and to the shared repository. This connector applies SPARQL Protocol and RDF Query Language (SPARQL) queries for ontology retrieval and upload. The functionalities and the user interface of the commercial package are kept in the background and the model of the production system is entirely defined in the ontology. As the simulation software does not possess a direct semantic reader a converter is required to correctly interpret the input data and their interrelations. This conversion is based on a specific XML table (represented as XML mapping in Figure 4.) which was also designed according to the ISA-95 standard.

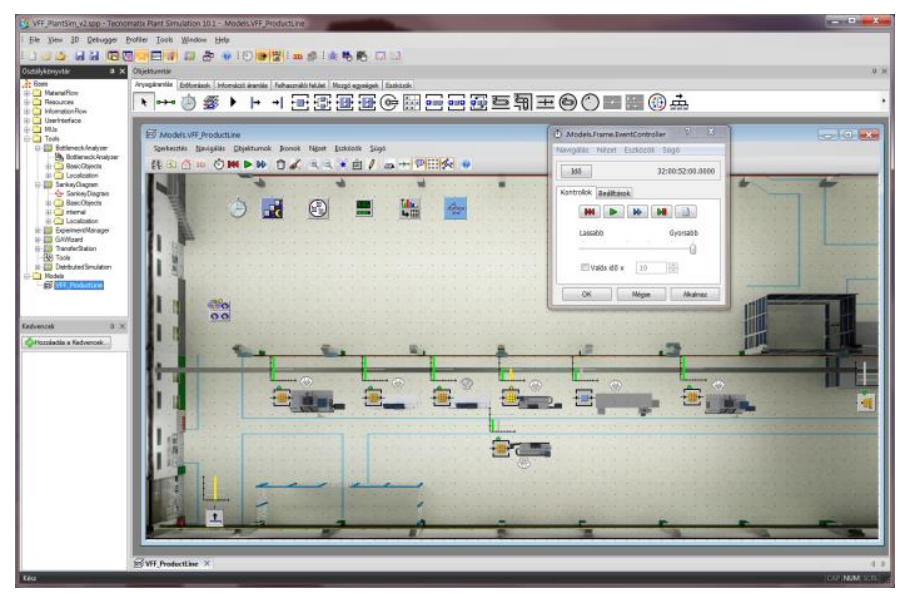

Figure 4.Representation of the test-case in Plant Simulation

The transformation of the model in the final, native Plant Simulation format is performed inside Plant Simulation in the SimTalk programming language. This process frees the user from manually creating the simulation model that is automatically generated from the initial VFDM-compliant factory project.

The creation of the simulation model is performed automatically as well as the initialization of the parameters and the model runs. In the current configuration the only data required by the user is the production order in a form of list indicating the products to be produced. A separate report and data visualization tool has been developed as well to support the presentation of simulation results. This is also achieved entirely through VFDM-based interoperation.

A similar approach can be adopted to integrate also other commercial DES tools into VFF, e.g. Arena as described in [22].

\section{Conclusion}

This paper has presented a framework to enable interoperability between software tools supporting the design and performance evaluation of a factory. The interoperability is based on a common semantic data model for representing virtual factories that was designed and implemented covering both the structural and operational aspects of production systems. custom-tailored or commercial tools can provide the same or subsequent functionalities required in the life-cycle of a manufacturing system. Further research should be carried out to develop more efficient solutions for accessing and managing huge amount of data in the shared repository and to exploit the enablers of the Semantic Web approach to perform reasoning and enrich the knowledge about specific manufacturing contexts.

\section{Acknowledgment}

The research reported in this paper has been funded by the European Union 7th FP (FP7/2007-2013) under the grant agreement No: NMP2 2010-228595, Virtual Factory Framework (VFF), the grant agreement No: 262044, VISION Advanced Infrastructure for Research (VISIONAIR) and National Office for Research and Technology (NKTH) grant "Digital, real-time enterprises and networks", OMFB-01638/2009.

\section{References}

[1] Terkaj, W.; Tolio, T.; Valente, A. (2009) Designing Manufacturing Flexibility in Dynamic Production Contexts, Design of Flexible Production Systems, Springer, Ch. 1:1-18.

[2] Monostori, L.; Erdős, G.; Kádár, B.; Kis, T.; Kovács, A.; Pfeiffer, A.; Váncza, J. (2010) Digital enterprise solution for integrated production planning and control, Computers in Industry 61(2):112-126.

[3] Maropoulos, P.G.: Digital enterprise technology - Defining perspectives and research priorities. In: Proc. of the 1st CIRP (UK) Sem. on Digital Enterprise Technology (DET02), September 16-17, 2002, Durham, UK, Part V: 3-12.

[4] Vernadat, F.B. (1994) Standards and prenorms in design, manufacturing and automation, Handbook of Design, Manufacturing and Automation (Dorf, R.C.; Kusiak, A. (Ed)), chapter 49:993-1019.

[5] Menzel, C.; Mayer R. J. (1998) The IDEF family of languages, Handbook on Architectures on Information Systems (Bernus, Mertins, Schmidt (Ed)), ch. 10:249-262.

[6] GERAM (1998) Generalised Enterprise Reference Architecture and Methodology, IFIP-IFAC Task force. ISO 15704 (ISO TC184/SC5/WG1 N423). Annex A.

[7] Scheer, A.W. (1999) ARIS- Business Process Modeling, Springer-Verlag, Berlins.

[8] ISO (2010) ISA-95: the international standard for the integration of enterprise and control systems. URL: http://www.isa-95.com/, last retrieved: October, 2012.

[9] National Institute of Standards and Technology. (2008) Process Specification Language (PSL). URL: http://www.mel.nist.gov/psl/, last retrieved: October, 2012.

[10] buildingSMART (2012). Industry Foundation Classes - IFC2x Edition 4 Release Candidate $2 . \quad$ URL: http://buildingsmarttech.org/ifc/IFC2x4/rc2/html/index.htm, last retrieved: October, 2012.

[11] Teo, Y. M.; Szabo, C. (2007): CODES: An Integrated Approach to Composable Modeling and Simulation, Asia Pacific Science and Technology Center SUN Microsystems Inc.

[12] Miller, J. A.; Baramidze, G. (2005) Simulation and the semantic web, Proceedings of the 2005 Winter Simulation Conference:2371-2377.

[13] Benjamin, P.; Patki, M.; Mayer, R. (2006) Using Ontologies for Simulation Modeling, Proc. of the 2006 Winter Simulation Conference: 1151-1159.

[14] Balci, O.; Arthur, J.; Ormsby, W. (2011) Achieving reusability and composability with a simulation conceptual model, Journal of Simulation 1(5):157-165.

[15] VFF (2012): VFF, Holistic, extensible, scalable and standard Virtual Factory Framework (FP7-NMP-2008-3.4-1, 228595). URL: http://www.vffproject.eu/, last retrieved: October, 2012.

[16] Sacco, M.; Dal Maso,G,; Milella, F.; Pedrazzoli, P.; Rovere, D.; Terkaj, W. (2011) Virtual Factory Manager in Lecture Notes in Computer Science, Ed: Springer:397-406.

[17] W3C (2004): OWL Web Ontology Language Reference, W3C Recommendation 10 Feb-ruary 2004. URL: http://www.w3.org/TR/owl-ref/, last retrieved October, 2012.

[18] Ghielmini, G., Pedrazzoli, P., Rovere, D., Terkaj, W., Dal Maso, G., Milella, F., Sacco, M., Boer, C.R. "Virtual Factory Manager of Semantic Data," in Proceedings of DET2011 7th International Conference on Digital Enterprise Technology, Athens, Greece, 2011

[19] W3C. (2004) RDF/XML Syntax Specification (Revised). [Online]. http://www.w3.org/TR/REC-rdfsyntax/ last retrieved December, 2012

[20] Terkaj, W; Pedrielli, G.; Sacco, M. (2012) Virtual Factory Data Model, Submitted to 2nd OSEMA (Ontology and Semantic Web for Manufacturing) Workshop Graz, Austria, July 24, 2012. [Online] http://ceur-ws.org/Vol886/paper_4.pdf.

[21] G.P. Viganò, L. Greci, S. Mottura, M. Sacco, (2011) GIOVE Virtual Factory: A New Viewer for a More Immersive Role of the User During Factory Design, in Digital Factory for Human-oriented Production Systems, L., Redaelli, C., Flores, M. Canetta, Ed.: Springer, 201-216.

[22] Terkaj W, Urgo M (2012) Virtual Factory Data Model to support Performance Evaluation of Production Systems. Proceedings of OSEMA 2012 Workshop, 7 th International Conference on Formal Ontology in Information Systems, Graz, Austria, 24-27 July, 2012. 